\title{
El caso del aplicativo móvil Uber frente al régimen de protección a la competencia en la legislación colombiana
}

\section{The case of the applied mobile Uber against the regime of protection to the competition in the Colombian legislation}

\section{Berónica Narváez Mercado ${ }^{1}$ Y Yeraldin Arrieta Ruiz² | Bertha Flores Gómez³ \\ 1 Doctorante de la Universidad Libre de Bogotá, Colombia. Correo electrónico: beronica.narvaez@cecar.edu.co \\ 2 Abogada de la Corporación Universitaria del Caribe, CECAR. Correo electrónico: yeraldin.arrieta@cecar.edu.co \\ 3 Maestrante en Derecho Procesal de la Universidad Simón Bolívar, Colombia. Correo electrónico: bertha.florezg@cecar.edu.co}

\section{RESUMEN}

El presente artículo analiza el régimen de protección de la competencia en la legislación colombiana, puntualmente en el caso Uber, una empresa prestadora del servicio público de transporte individual de pasajeros, a través de un aplicativo tecnológico. Se desarrolló una investigación de corte cualitativo, jurídico y hermenéutico, donde se utilizaron fuentes de información secundarias para resolver el caso objeto de debate, con el objetivo de determinar el régimen de protección a la competencia, la modalidad bajo la cual opera, Uber, la empresa desarrolladora del software, y los entes nacionales encargados de su vigilancia y control, logrando concluir que de acuerdo a la naturaleza esencial del servicio prestado, es el Estado, a través del Ministerio de Transporte y la Superintendencia de Puertos y Transportes, el ente encargado de su vigilancia y control y que Uber no incurre en ninguna competencia desleal frente al servicio tradicional de taxi, a la luz de la legislación colombiana vigente.

Palabras clave: Aplicativo tecnológico, competencia desleal, régimen de competencia y Uber.

\section{ABSTRACT}

This article analyzes the regime of protection of competition in Colombian legislation, punctually in the case of Uber, a company that provides the public service of individual passenger transport, through a technological application. A qualitative, juridical and hermeneutical investigation was developed, where secondary sources of information were used to solve the case under discussion, with the objective of determining the regime of protection to the competition, the modality under which the company Uber develops the software and the national entities in charge of monitoring and control of the same, managing to conclude that according to the essential nature of the service provided, it is the State, through the Ministry of Transport and the Superintendence of Ports and Transport, the entity in charge of its monitoring and control and that Uber does not incur any unfair competition against the traditional taxi service, in light of current Colombian legislation. Key words: Technological application, unfair competition, regime of competition and Uber.

\section{JUSTICIA}

Recibido: 04-05-17 Aceptado: 03-09-17 Publicado: $18-01-18$

Dol: 


\section{INTRODUCCIÓN}

La entrada de muchas empresas que prestan sus servicios a través de aplicativos móviles hoy en día, es una realidad a la que ningún país puede escapar. Las necesidades de las comunidades van evolucionando y las dinámicas del mercado se van globalizando. Es así como surgen cambios en los modelos de mercados, los cuales deben ir ajustándose a las políticas internas de los Estados y a su reglamentación o normatividad. Se realizó un estudio del régimen de protección a la competencia en Colombia, mirada desde ese paradigma histórico de enfrentamiento de las empresas por la clientela. Estrategias que buscan los empresarios para atraer usuarios e incrementar su patrimonio, constantes luchas por mostrar o lograr la confianza de las personas y de esta forma obtener la preferencia en el mercado.

El mercado analizado fue el de la prestación del servicio público de transporte individual de personas, el cual está en cabeza del Estado su vigilancia y control, de acuerdo a la Ley 336 de 1996, dinámicas de prestación del servicio que han cambiado y los grandes empresarios han buscado la adquisición y diseño de nuevas metodologías para este fin, como la implementación de software $u$ aplicativos móviles, innovaciones que son de esperarse en pleno siglo XXI.

Surge hacia el año 2009 la empresa Uber, la cual funciona con la instalación de un aplicativo móvil, sirviendo de intermediaria para prestar el servicio de transporte individual de pasajeros, como lo sostiene Hernández \& Galindo (2016, p. 164), y ha tenido gran acogida por parte de los consumidores en muchos países. Está claro que los empresarios deben adoptar sus estrategias competitivas a las Tecnologías de la Información y las Comunicaciones, pero no dejando de lado la protección al principal miembro de la cadena de consumo: el consumidor, protagonista en todo este proceso de fortalecimiento en los mercados.

Es lo que sostiene Lucas Llina (2017), gerente general de operaciones de Uber en Colombia, quien afirma que, precisamente lo que buscan es mejorar la calidad de vida de las personas y contribuir con los desafíos de la movilidad en las ciudades. Pero por otro lado están presentes viejos monopolios como lo afirma Granados (2014), donde son los dueños de los "taxis amarillos", los que están en contra de la aplicación móvil, toda vez que son estos los que mayor lucro obtienen de la circulación de dichos vehículos en las ciudades colombianas, manifestando inconformidades por una presunta competencia desleal. 
Es por lo que se decidió analizar la prestación del servicio público de transporte individual, prestado a través de esta aplicación tecnológica, que se ha inmiscuido en el mercado colombiano con más fuerza, desde el año 2015 en las principales ciudades como Bogotá, Medellín, Cali y Barranquilla. Aplicación, que surge inicialmente en el comercio estadounidense, específicamente en San Francisco California, como una forma de mostrar una alternativa a la demanda de pasajeros en dicho territorio; posteriormente expandiéndose en diferentes ciudades del mundo como las del continente europeo, asiático y americano (Sánchez, Coronel \& Castellanos, 2014, p. 19).

El objeto principal de estudio mostrado en este artículo es el caso colombiano, el cual ha traído muchas controversias desde que entró en funcionamiento dicha aplicación, debido a que los gremios de taxistas han realizado paros, como muestra de rechazo a la entrada en circulación de estos carros, muchos de ellos de lujo y que ofrecen unos servicios considerados de primera línea, frente al servicio prestado por un taxi normal en Colombia.

Por lo que se han pronunciado respecto al caso varios entes nacionales como la Superintendencia de Industria y Comercio (2015), la Superintendencia de Puertos y Transportes (2016), el Ministerio de las Tecnologías de la Información y las Comunicaciones (2017), junto con la Agencia Nacional del Espectro y el Ministerio de Transporte (2017), algunos de estos para defender la postura de abrir el mercado colombiano a las nuevas tecnologías y otros para rechazar esta forma de prestación de servicio, la cual no tiene una regulación en el ordenamiento jurídico interno.

Por ello la investigación se orientó bajo la siguiente pregunta problema: ¿Cuál es la aplicabilidad del régimen de protección de la competencia en la legislación colombiana, en el caso del servicio de transporte Uber? Frente a ella se desprenden problemáticas que también son objeto de análisis como es el caso muy puntual sobre el estudio de la configuración de una competencia desleal con este tipo de transporte.

Como objetivo general en este estudio se trazó: Determinar el régimen de protección de la competencia en la legislación colombiana, en el caso del servicio de transporte Uber; a su vez para alcanzar la consecución de este gran derrotero se trazaron los siguientes objetivos específicos: I. Identificar el marco normativo aplicable a la empresa de tecnología y servicio de transporte Uber en Colombia. II. Establecer en qué actos de competencia desleal previstos en la legislación colombiana incurre la empresa de tecnología Uber 
III. Determinar el ente competente para el control y vigilancia, de la empresa Uber en Colombia de acuerdo a la figura o modalidad bajo la cual opera.

Es importante resaltar la pertinencia de indagar sobre este tema de actualidad porque cada día va en aumento el número de relaciones comerciales que son realizadas a través de dispositivos móviles, utilizando las Tecnologías de la Información y las Comunicaciones. De igual forma, la concentración de población que se acentúa en cada ciudad capital es mayor, por lo que el sistema de transporte público no logra cubrir la demanda de usuarios que requieren de dicho servicio. De ahí, el surgimiento de otras opciones como el mototaxismo en la costa Caribe colombiana o la entrada de servicios como Uber y Cabify al mercado nacional, empresas innovadoras prestadoras del servicio individual de transporte a través de dispositivos móviles y con servicios considerados de lujo.

\section{APLICACIÓN UBER EN EL MERCADO INTERNACIONAL Y NACIONAL}

Uber es una compañía desarrolladora de software, la cual tuvo sus inicios en San Francisco (Estados Unidos), específicamente presta el servicio público individual de transporte en automóviles particulares que ofrecen un servicio especial. Todos los conductores que trabajan para este sistema se inscriben a través de la plataforma web diseñada para tal fin, a los cuales se les verifican sus antecedentes antes de vincularse a laborar y una vez pasado el examen, deben pagar un porcentaje por cada una de las carreras que realicen.

Esta aplicación se expandió más allá de las fronteras norteamericanas, haciendo presencia hoy en día en más de cincuenta ciudades importantes en todo el mundo. Solo de imaginar que se puede tener a la mano de una forma muy sencilla, ágil y en contados minutos el servicio de un automóvil modelo 2009 en adelante, eso hoy es posible a través de la aplicación Uber, cuya empresa ya ha entrado a competir en el mercado colombiano. Uber hoy en día es legal en Inglaterra y Gales, los cuales hacen parte del Reino Unido, por medio de un fallo de la Alta Corte, donde manifestó que este programa informático no utiliza los sistemas de taxímetro a la hora de establecer el valor de un viaje, al contrario, requiere de una señal GPS. De igual forma en Broward, Florida (Estados Unidos) se aprobó una moción que legaliza el servicio según Infobae (2015). En Latinoamérica ha ganado legalmente en Brasil después de varias luchas, como lo dio a conocer la Agencia EFE (2016), donde se le concedió laborar por medio de medidas cautelares. La Alcaldía de Sao Paulo expidió un Decreto donde regula la aplicación, permitiendo operar en la 
metrópolis, debiendo pagar a la administración una tasa mensual para poder operar, más otros requisitos que debe cumplir.

En Colombia el gremio de los taxistas, en varias ocasiones ha salido a protestar a las calles, rechazando el modo de operar de Uber, debido a que argumentan que es una clara competencia desleal, sostienen que se les ha permitido operar hasta este momento sin estar reconocidos como una empresa de transporte. Lo anterior les permite poder ofrecer el servicio público de lujo, sin necesidad de cancelar el valor de un cupo, el cual, si debe cancelar el propietario de un taxi, tampoco los impuestos y pólizas que debe sufragar un taxista.

Por su parte, el Ministerio de Transporte expidió la Resolución 2163 de 2016, por medio de la cual reglamentó el Decreto 2297 de 2015, este último delimita los parámetros bajo los cuales debe funcionar el servicio de lujo, dentro de la modalidad de servicio público de transporte individual. Serían en principio, y de acuerdo a la naturaleza del servicio prestado, el Ministerio de Transporte y la Superintendencia de Puertos y Transporte los entes a nivel nacional encargados de la vigilancia, control y supervisión de todas las empresas que presten el servicio público de transporte en Colombia.

Hasta este punto la normatividad vigente se encargó de reglamentar el servicio de transporte de lujo de pasajeros y todo el procedimiento que deben cumplir ante el Ministerio de Transporte, para entrar a operar como cualquier otro vehículo que a nivel nacional preste el servicio. Por otro lado, exige que las herramientas tecnológicas empleadas deben ser vinculadas al servicio público de transporte, en otras palabras, los softwares implementados deben hacer parte de la empresa que preste dicho servicio y no estar constituidas como simple intermediarios, según el Decreto 2060 de 2015, por medio del cual se reglamentaron los sistemas inteligentes en el país.

De acuerdo al análisis realizado sobre la normativa al respecto, se puede decir que se quedó corta en cuanto muchos aspectos de carácter particular, como la diferencia pecuniaria que deben sufragar los taxis al momento de cancelar el valor de cupos, seguros $u$ otros tipos de reglamentaciones que deben cumplir las personas que prestan el servicio público de taxi. De acuerdo con el Decreto 2297 de 2015 y la Resolución 2163 de 2016, que reglamentan y permiten que en el mercado presten el servicio público de transporte individual de pasajeros, automóviles de lujo y que puedan utilizar plataformas web, para mejorar el servicio. 


\section{RÉGIMEN DE PROTECCIÓN DE LA COMPETENCIA Y ACTOS DE COMPETENCIA DESLEAL A LA LUZ DE LA LEGISLACIÓN COLOMBIANA}

La libre competencia económica es un derecho colectivo, consagrado en la Constitución Política, en el artículo 333, el cual les otorga a todas las personas facultades para realizar actividades económicas de carácter privado, siempre y cuando no afecten el bien común, lo que implica responsabilidades por parte de todas las personas que tengan este tipo de iniciativas.

A través de todo el desarrollo legal de la competencia en Colombia, se han creado diferentes normas para regularlo, tales como la Ley 155 de 1959, la primera en entrar a regular el régimen de competencia, pero que no era clara en cuanto a quién era el ente encargado de tal situación, naciendo tiempo después el Decreto 2153 de 1992, el cual fijó la política de la competencia en el país. Surgiendo diecisiete años después la Ley 1340 del año 2009, para regular las nuevas necesidades del mercado y la protección al consumidor, otorgándole a su vez a la Superintendencia de Industria y Comercio la responsabilidad, como la única autoridad encargada de aplicar en todos los sectores de la economía normas de competencia, según lo estableció la Organización para la Cooperación y el Desarrollo Económicos (OCDE, 2009).

Ahora en este artículo se analiza lo concerniente a la protección de la competencia, al respecto se puede decir que la entidad exclusiva y encargada de velar por la cabal aplicación de las normas que la regulan lo ha definido como:

El derecho de competencia es aquel que se ocupa de la protección de los intereses de los consumidores y de la protección de la libre competencia en los mercados, mediante la prohibición de actos que se consideran impiden la competencia y mediante la promoción y abogacía por un entorno competitivo (Superintendencia de Industria y Comercio, 2016).

Se hace necesario reiterar en este punto, que el objeto de nuestra investigación es la libre competencia y a su vez, cuáles son los actos prohibidos o que violan este régimen. Es por lo que se reafirma que inicialmente este derecho está en cabeza de todas las personas que deseen tomar la iniciativa de crear, organizar y emprender su propia actividad económica, precisamente sin vulnerar los límites propios establecidos en la legislación para ello, es decir, es un derecho de carácter colectivo.

Pero también es importante establecer cuáles son los límites a este derecho, 
y precisamente la Ley 1340 de 2009 trae un catálogo no exhaustivo de conductas que se consideran que atentan contra el derecho constitucional a la libre competencia. Surge la necesidad de limitarlo y es precisamente lo que previó el constituyente y el legislador, como forma de garantizar a todos los consumidores y personas que tienen una actividad económica debidamente constituida, para que a la hora de ejercerla no realicen conductas que afecten las libres y sanas prácticas de mercado, las cuales entrarían no solo a beneficiar a los que ostentan la posición dominante, sino al consumidor como miembro débil de la cadena de consumo.

Entre todo este listado de conductas nos interesa la que establece la Ley 256 de 1996 en el capítulo II, respecto a los actos de competencia desleal. Soportado en la buena fe comercial y precisamente esa lealtad entre las competencias del mercado. Una vez analizados los doce artículos que hacen referencia a los actos de competencia desleal en la normatividad vigente colombiana, se pudo identificar que el artículo 18, en cuanto a la violación de normas, es el que más se acerca a un posible acto de competencia desleal, cometido por la empresa Uber. Dicho artículo reza de la siguiente manera: "se considera desleal la efectiva realización en el mercado de una ventaja competitiva adquirida frente a los competidores mediante la infracción de una norma jurídica. La ventaja ha de ser significativa".

Uber goza de una ventaja competitiva adquirida frente a las empresas que prestan el servicio tradicional de taxis en el país, toda vez que, por ejemplo, para entrar a transportar un taxi debe adquirir un tarjeta de operación, que se oficializa por parte de la autoridad de transporte competente anualmente, la cual debe obtener el Seguro Obligatorio de Accidentes de Tránsito (SOAT), la licencia de tránsito de vehículos, revisión técnico-mecánica vigente del vehículo, pólizas de responsabilidad civil contractual y extracontractual de la empresa y acreditar la consignación a favor de la autoridad de transporte competente por el pago de los derechos que se causen, todos estos requisitos según el Decreto 172 de 2001, por medio del cual se reglamentó el servicio público de transporte terrestre automotor individual de pasajeros en vehículos taxis.

Desde este punto de vista, se puede afirmar que los conductores que prestan el servicio público de transporte individual de personas por medio de taxis tradicionales tienen una desventaja notoria frente al transporte al servicio prestado por los automóviles catalogados como de lujo; como se viene sosteniendo en párrafos anteriores. Sin embargo, la ventaja competitiva no es 
adquirida mediante la infracción de una norma jurídica, tal como lo establece la normatividad y no podría ser catalogada como una competencia desleal.

Toda vez que el transporte de lujo de pasajeros lo pueden prestar todas las empresas o personas en el país, que cumplan con los requisitos establecidos en la normatividad vigente para ello, dicho servicio no está restringido solamente para aplicativos móviles o software como el que posee Uber a nivel internacional, lo que deja abierta la puerta para que nuevos inversionistas, comerciantes y empresarios puedan entrar a competir bajo esta modalidad, de prestación del servicio en Colombia.

A pesar de existir inconformismos por parte de las empresas que han prestado el servicio tradicional de transporte individual de personas, los cuales han querido que los entes nacionales excluyan o prohíban la operación de la empresa Uber en el mercado nacional, no se vislumbra por esta última la intención de fragmentar el principio de buena fe comercial que pregona nuestro legislador, toda vez que hasta este momento no se estaría infringiendo ninguno de los actos de competencia desleal estatuidos en nuestro ordenamiento jurídico.

\section{ENTE COMPETENTE PARA REGULAR LA APLICACIÓN DE SERVICIOS DE TRANSPORTE COMO UBER}

Al hablar del servicio de transporte público en Colombia los entes competentes para regularlo son el Ministerio de Transporte y la Superintendencia de Puertos y Transportes y los encargados de llevar el control, vigilancia y seguimiento de las empresas dedicadas al transporte de personas en nuestro país a nivel nacional. Y a nivel municipal y distrital estarían los Alcaldes Municipales o Distritales o los organismos que estos deleguen, de acuerdo al artículo 8 del Decreto 172 de 2001. Pero si, por el contrario, se define a Uber como ellos mismos se autodenominan como empresa tecnológica, intermediaria entre conductores particulares y pasajeros, entraría a jugar un papel importante para diseñar, adoptar y promover las políticas, planes y programas del sector de las Tecnologías de la Información y las Comunicaciones, el Ministerio de las Tecnologías de la Información y las Comunicaciones y la Agencia Nacional del Espectro (ANE) (Ministerio de las TIC, 2016).

Este último concepto, por el cual viene operando Uber en Colombia, sería un poco más alejado de la realidad comercial, debido a que el servicio principal que prestan a través de la plataforma es el de transporte público individual de personas, siendo la principal actividad económica que les comercializa la 
plataforma a los usuarios, aunque no se puede desconocer que estarían en juego elementos muy importantes que están siendo explotados, como lo es el espectro electromagnético, el cual es un bien de uso público.

Precisamente, el debate en todo el entorno de las controversias surgidas con la entrada en el mercado colombiano, es el de la libre competencia frente a la competencia desleal, esta última tiene como órgano encargado de la vigilancia y control a la Superintendencia de Industria y Comercio, ente que a pesar de tener esta función en los temas concernientes a la libre competencia económica, no sería el encargado en el caso puntual, debido a que como se ha venido analizando Uber no ha venido infringiendo normas que afecten la competencia, frente al sistema prestado por los taxis tradicionales en el país. Por el contrario, el mercado colombiano debe reglamentar y actualizar su normatividad a las nuevas tendencias internacionales, para buscar el equilibrio social y económico en la prestación del servicio de transporte en sus localidades, que no está actualmente reglamentado para este tipo de empresas.

A nivel nacional, se han presentado demandas en contra de Uber, sosteniendo que debe ser bloqueada la plataforma, toda vez que se lucra y explota el espectro electromagnético colombiano sin permiso; postura que es apoyada por el Ministerio de Transporte. Por otro lado, el Ministerio de Tecnologías de la Información y las Comunicaciones defiende la aplicación, sosteniendo que hasta la fecha en Colombia no se restringido ninguna aplicación que utilice el espectro electromagnético, solamente se han prohibido cuando se trate de contenidos sobre pornografía infantil; porque de ser así manifiesta que existen aplicaciones como WhatsApp, Netflix y Airbnb, solo por mencionar algunas, a las que también se debería reglamentar por la utilización de tal bien de uso público (Revista Semana, 2017).

Sigue sosteniendo el Ministerio de Tecnologías de la Información y las Comunicaciones, que de inhabilitar la plataforma se estaría atentando contra el principio internacional de neutralidad de red, que va de la mano del derecho fundamental a la libertad de expresión. Este derecho internacional ha tenido bastante desarrollo legal y doctrinal en varios Estados, el cual hace referencia, como sostiene Espinosa (2009, p. 87), a la infraestructura lógica y económica de los servicios y productos informáticos. En otras palabras, este principio actualmente pasa por interesantes debates sobre la regulación económica del uso de la tecnología, siendo el Internet hoy en día una herramienta que satisface necesidades colectivas. 
Le corresponde al Estado llenar el vacío del Derecho, comenzando a reinventar y reivindicar el caos generador de una conversación mundial sin fin, en palabras de Espinosa (2009, p. 127), tal cual como está sucediendo en la actualidad social con la empresa Uber, que está prestando el servicio de transporte individual de pasajeros a través de un aplicativo tecnológico. Pero mañana puede ser otro u otras empresas que desarrollen aplicativos, software o programas e incursionen en el mercado nacional e internacional.

Actualmente la Ley 1341 de 2009 efectivamente protege la neutralidad tecnológica y de la red en nuestro medio, pero de igual forma les impone a todos aquellos particulares la carga de pagar una contraprestación económica a quienes hagan uso del espectro radioeléctrico en el país y define el proceso para acceder a dichos permisos. He aquí donde le corresponde al Estado entrar a regular en el caso puntal, estrategias para equilibrar este tipo de sucesos en el mercado de transporte en el país, debido a que tecnológicamente de acuerdo con las leyes vigentes de protección del uso de plataformas electrónicas Uber y otras empresas que presten servicios por medio de aplicativos de este tipo son legales. Solo que el Estado deberá intervenir para proteger a todos los consumidores y demás miembros de la cadena de consumo para equilibrar las dinámicas del mercado.

Será el Estado, a través del Ministerio de Transporte, inicialmente, la entidad encargada de dar las especificaciones y condiciones bajo las cuales operarían las empresas prestadoras del servicio público de transporte, sea básico o de lujo. Una vez definidos todos los lineamientos, serán responsables del seguimiento y cumplimiento de estos parámetros, junto con la Superintendencia de Puertos y Transportes, para exigir el acatamiento de las normas creadas para tal regulación. Por último, en los casos donde se susciten casos de competencia desleal, ahí sí entraría la Superintendencia de Industria y Comercio a dirimir el asunto en concreto, para buscar la igualdad en el mercado y proteger a los consumidores y todos los miembros de la cadena de consumo. Pero esto último, una vez que el Estado intervenga a regular por completo con medidas efectivas los casos del servicio de transporte individual de personas a través de aplicativos móviles.

\section{RESULTADOS}

De los resultados arrojados por la investigación se pudo determinar que el régimen de protección de la competencia en la legislación colombiana, para resolver las controversias surgidas por la empresa tecnológica Uber y el sistema de taxis tradicionales, no sería la correspondiente en materia 
comercial a la Ley 256 de 1996 y Ley 1340 de 2009, en las cuales entraría a dirimir los conflictos en la materia la Superintendencia de Industria y Comercio a nivel nacional. Tal cual como está establecido para aquellos casos donde se presenten debates y afectaciones a la sana competencia en el mercado.

Sería entonces en principio el Gobierno Nacional, a través del Ministerio de Transporte y la Superintendencia de Puertos y Transportes, los entes encargados de velar por la normal y adecuada prestación del servicio público e individual de transporte de personas en el país; así como de garantizar la igualdad entre las distintas empresas y modalidades que compitan en el mercado.

\section{CONCLUSIÓN}

El marco normativo aplicable a la empresa de tecnología y servicio de transporte Uber en Colombia, sería en la actualidad la Resolución 2163 de 2016, que reglamentó el Decreto 2297 de 2015. Los cuales buscan reglamentar el servicio de transporte de lujo en el país, en otras palabras, buscan que las empresas dedicadas al transporte individual de pasajeros, en la categoría de lujo, adquieran las plataformas desarrolladoras de software como Uber y las hagan parte de la empresa de transporte, pero sin prever los temas de seguros, pólizas e impuestos que deben pagar las empresas que operen bajo esta modalidad especial.

Es por ello que el Ministerio de las Tecnologías de la Información y las Comunicaciones entraría a proteger la utilización del espacio radioeléctrico, tal como lo designa la Ley 1341 de 2009, bajo la cual se le impone la obligación de velar y otorgar permisos para la utilización de este bien público, sobre todo el territorio nacional.

De acuerdo a los actos de competencia desleal contemplados por la legislación colombiana, la empresa Uber no infringe ningún acto que afecte la sana competencia en el mercado nacional. Una vez realizado un minucioso análisis de cada uno de los actos contemplados por la normatividad vigente. No desconociendo las claras desventajas que se presentan hasta el momento, en los temas relacionados a la prestación del servicio y el pago de contraprestaciones económicas. Por un lado la seguridad del pasajero en temas contractuales y por el otro, el pago por la utilización de ciertos bienes de uso público al Estado.

El ente competente a nivel nacional para el control y vigilancia de los servicios 
de transporte individual de pasajeros, en la modalidad de lujo y con la utilización de plataformas tecnológicas será el Estado a través del Ministerio de Transportes y la Superintendencia de Puertos y Transportes, en razón al servicio público y esencial que prestan a la comunidad.

\section{REFERENCIAS BIBLIOGRÁFICAS}

Agencia EFE (10 de 05 de 2016). Edición América - Economía. Obtenido de Regulan el uso de la aplicación Uber en Sao Paulo: http://www.efe. com/efe/america/economia/regulan-el-uso-de-la-aplicacion-uber-en sao-paulo/20000011-2922014

\section{Normativa nacional}

Colombia. Constitución Política de Colombia. Art. 333. Julio 7 de 1991.

Colombia. Decreto 2153 de 1992 [Ministerio de Desarrollo Económico]. Por el cual se reestructura la Superintendencia de Industria y Comercio. Diciembre 30 de 1992.

Colombia. Decreto 172 de 2001 [Ministerio de Transporte]. Por el cual se reglamenta el Servicio Público de Transporte Terrestre Automotor Individual de Pasajeros en Vehículos Taxi. Febrero 05 de 2001.

Colombia. Decreto 2060 de 2015 [Ministerio de Transporte]. Por el cual se adiciona el Decreto 1079 de 2015 y se reglamenta el artículo 84 de la Ley 1450 de 2011. Octubre 22 de 2015.

Colombia. Decreto 2297 de 2015 [Ministerio de Transporte]. Por el cual se modifica y adiciona el Capítulo 3, Título 1, Parte 2, Libro 2 del Decreto número 1079 de 2015, en relación con la prestación del servicio público de transporte terrestre automotor individual de pasajeros en niveles básico y de lujo. Noviembre 27 de 2015.

Colombia. Ley 1340 de 2009. Por la cual se dictan normas en materia de protección de la competencia. Julio 24 de 2009. DO. №47420

Colombia. Ley 1341 de 2009. Por la cual se definen principios y conceptos sobre la sociedad de la información y la organización de las Tecnologías de la Información y las Comunicaciones -TIC-, se crea la Agencia Nacional de Espectro y se dictan otras disposiciones. Julio 30 de 2009. DO. N47426

Colombia. Ley 155 de 1959. Por la cual se dictan algunas disposiciones sobre prácticas comerciales restrictivas. Diciembre 24 de 1959. DO. $N^{\circ} 30138$

Colombia. Ley 256 de 1996. Por la cual se dictan normas sobre competencia desleal. Diciembre 20 de1996. DO. N42948

Colombia. Ley 336 de 1996. Por la cual se adopta el Estatuto Nacional de Transporte. Enero 15 de 1996. DO. N42692

Colombia. Resolución 2163 de 2016 [Ministerio de Transporte]. Por la cual se Reglamenta el Decreto 2297 de 2015 y se dictan otras disposiciones. Mayo 27 de 2016. 


\section{Textos}

Agencia Nacional del Espectro y el Ministerio de Transporte (2017). ¿Por qué peligra la plataforma de Uber? (4 de enero de 2017). Recuperado de http://www.semana.com/nacion/articulo/demanda-del-ministerio-de -transporte-a uber/520506

Espinosa, C. (2009). La información en la red y el principio de neutralidad tecnológica: la libertad de expresión y la difusión de información admisnistrativa. En. Revista Derecho del Estado, 83-128.

Granados, N. S. (05 de octubre de 2014). UBER presiona al monopolio amarillo. El Libre Pensador (24).

Hernández \& Galindo, Y. R. (2016). Modelo de gestión del servicio de transporte UBER. ¿Quién pierde y quién gana? Espacios Públicos. Universidad Autónoma del Estado de México, 164.

Infobae América (16 de octubre de 2015). La Corte inglesa dictaminó que Uber es legal en Londres. Obtenido de http://www.infobae. com/2015/10/16/1762764-la-corte-inglesa-dictamino-que-uber-es-legal-londres/

Linda Salazar Sánchez, S. J. (2014). Uber: ¿un negocio innovador desregularizado o vulnerador de la libre competencia? Justicia y Derecho, 7-39.

Llina, L. (05 de mayo de 2017). La regulación no puede definirse en favor de viejos monopolios, gerente de Uber. Obtenido de Dinero: http://www.dinero.com/empresas/articulo/ uber-y-los-retos-para-legalizar-en-colombia/245508

MINTIC (26 de 08 de 2016). Ministerio de las Tecnologías de la Información y las Comunicaciones. Obtenido de http://www.mintic.gov.co/portal/604/ w3-propertyvalue-540.html

MINTIC (2017). Ministerio de las Tecnologías de la Información y las Comunicaciones. Demanda del Mintransporte: no bloqueará Uber (23 de marzo de 2017). Recuperado de http://www.enter.co/cultural-digital/ colombia-digital/mintic-respondio-a-demanda-del-mintransporte-no -bloqueara-uber/

OCDE (2009). Derecho y política de competencia en Colombia. Obtenido de Organización de Cooperación y Desarrollo Económicos: https://www. oecd.org/countries/colombia/44111213.pdf

Revista Semana (04 de enero de 2017). ¿Por qué peligra la plataforma Uber? Obtenido de http://www.semana.com/nacion/articulo/ demanda-del-ministerio-de-transporte-a-uber/520506

Sánchez, S., Coronel, Y. \& Castellanos, L. (2014). Uber: ¿Un negocio innovador desregularizado o vulnerador de la libre competencia? En. Bepress. Recuperado de https://works.bepress.com/carlospg94/15/, p. 19.

SIC (2015). Superintendencia de Industria y Comercio. Obtenido de ¿Qué es la protección de la competencia?. http://www.sic.gov.co/drupal/ que-es-la-proteccion-de-la-competencia

Supertransporte (2016). Superintendencia de Puertos y Transportes. Sanción impuesta a Uber Colombia S.A.S. En. Revista Semana (31 de 
octubre de 2016). Recuperado de http://www.semana.com/tecnología/ artículo/superintendencia-de-puertos-y-transportes-amenaza-con-multas-a-uber/491987

Uber (2017). Requisitos para manejar con Uber. Obtenido de https://www. uber.com/es-CO/drive/requirements/ 